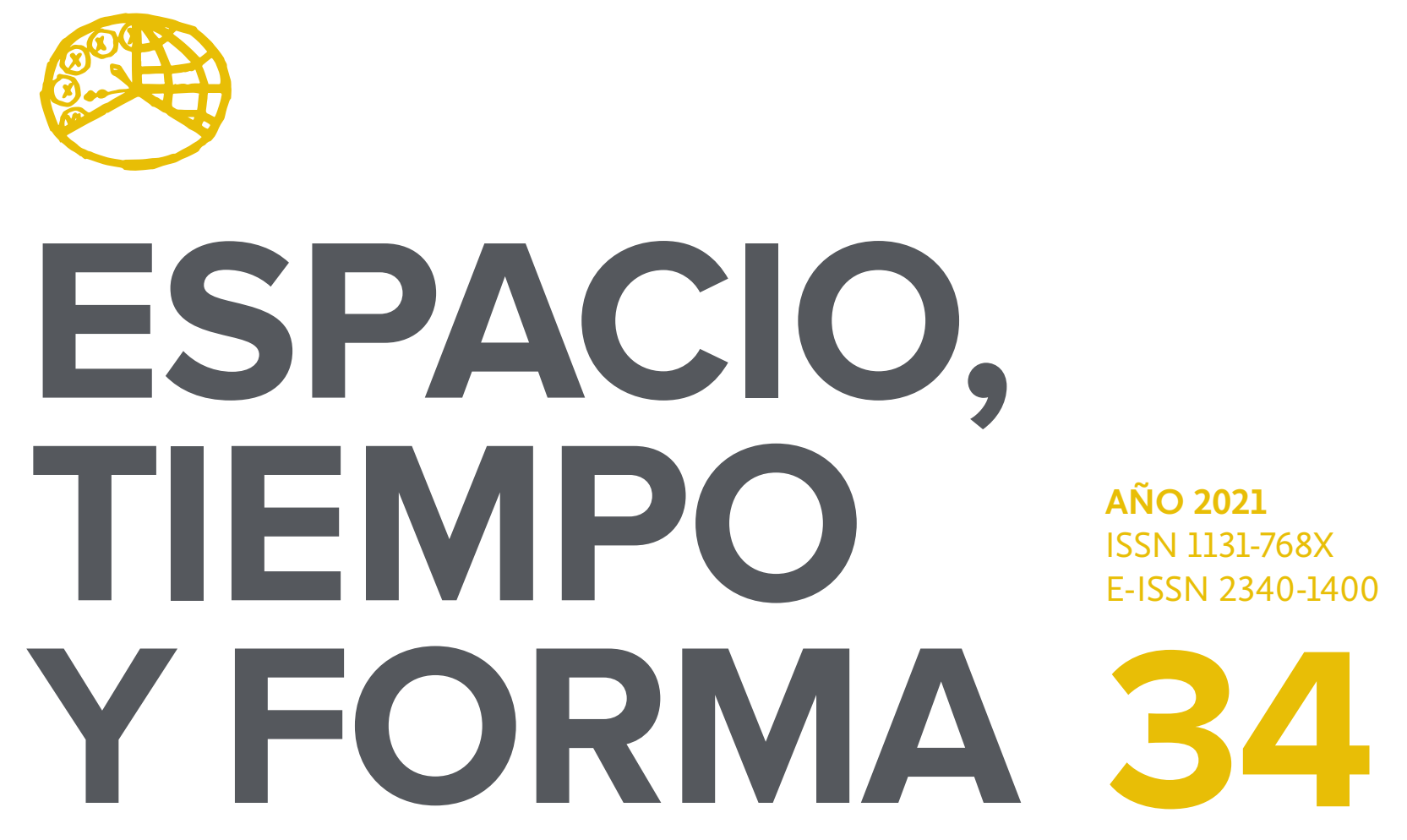

SERIE IV HISTORIA MODERNA

REVISTA DE LA FACULTAD DE GEOGRAFÍA E HISTORIA

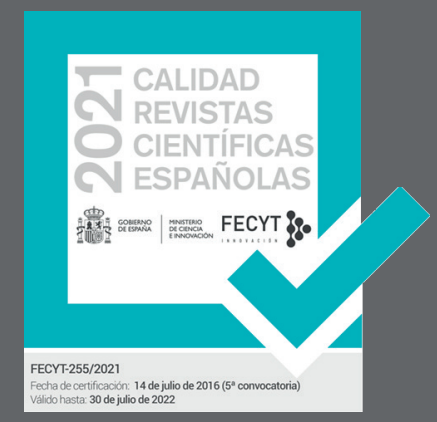




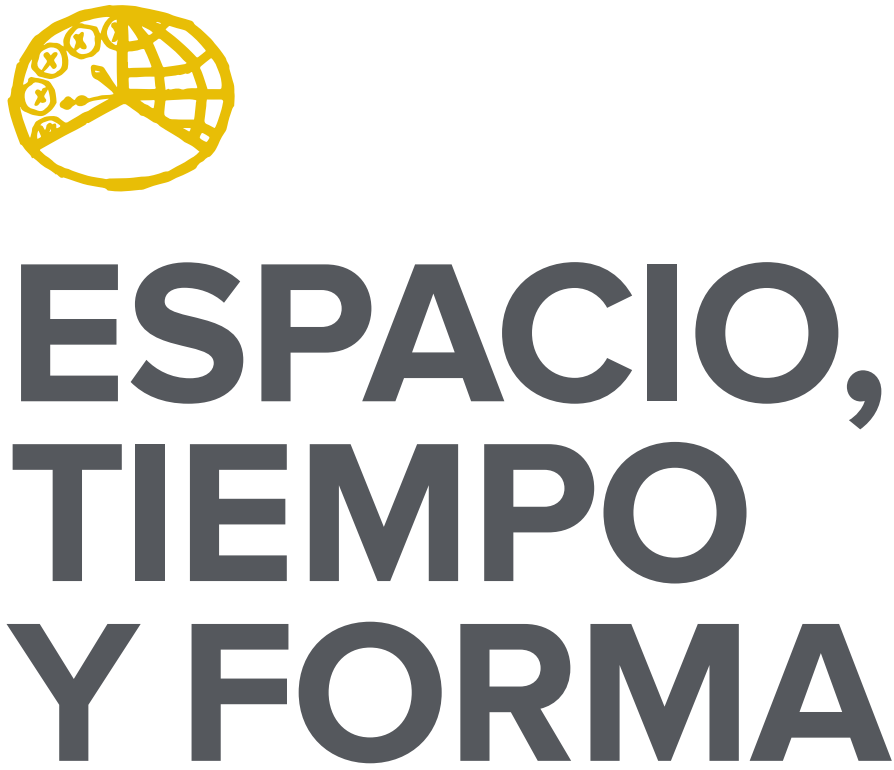

AÑO 2021

ISSN 1131-768X

E-ISSN 2340-1400

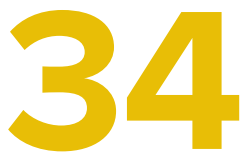

SERIE IV HISTORIA MODERNA

REVISTA DE LA FACULTAD DE GEOGRAFÍA E HISTORIA

DOI: https://doi.org/10.5944/etfiv.34.2021

\section{UกED}

UNIVERSIDAD NACIONAL DE EDUCACIÓN A DISTANCIA 
La revista Espacio, Tiempo y Forma (siglas recomendadas: ETF), de la Facultad de Geografía e Historia de la UNED, que inició su publicación el año 1988, está organizada de la siguiente forma:

$$
\begin{aligned}
& \text { SERIE I - Prehistoria y Arqueología } \\
& \text { SERIE II - Historia Antigua } \\
& \text { SERIE III - Historia Medieval } \\
& \text { SERIE IV - Historia Moderna } \\
& \text { SERIE V - Historia Contemporánea } \\
& \text { SERIE VI - Geografía } \\
& \text { SERIE VII - Historia del Arte }
\end{aligned}
$$

Excepcionalmente, algunos volúmenes del año 1988 atienden a la siguiente numeración:

$$
\begin{aligned}
& \mathrm{N} .^{\circ} 1 \text { - Historia Contemporánea } \\
& \mathrm{N}^{\circ} 2 \text { - Historia del Arte } \\
& \mathrm{N} .^{\circ} 3 \text { - Geografía } \\
& \mathrm{N} .^{\circ} 4 \text { - Historia Moderna }
\end{aligned}
$$

ETF no se solidariza necesariamente con las opiniones expresadas por los autores.

\author{
UNIVERSIDAD NACIONAL DE EDUCACIÓN A DISTANCIA \\ Madrid, 2021 \\ SERIE IV · HISTORIA MODERNA N. ${ }^{\circ} 34,2021$ \\ ISSN $1131-768 X \cdot$ E-ISSN 2340-1400 \\ DEPÓSITO LEGAL \\ M-21.037-1988 \\ URL \\ ETF IV · HISTORIA MODERNA · http://revistas.uned.es/index.php/ETFIV \\ DISEÑO Y COMPOSICIÓN \\ Carmen Chincoa Gallardo · http://www.laurisilva.net/cch \\ Impreso en España · Printed in Spain
}

(c) (7) (8) Esta obra está bajo una licencia Creative Commons Reconocimiento-NoComercial 4.0 Internacional. 


\section{TALLER DE HISTORIOGRAFÍA .}

HISTORIOGRAPHY WORKSHOP

ENSAYOS - ESSAYS 



\title{
LAS RELACIONES HISPANO-CHINAS EN EL SIGLO XVI: SÍNTESIS E INTERPRETACIÓN A LA LUZ DE LA RECIENTE HISTORIOGRAFÍA
}

\section{THE HISPANIC-CHINESE RELATIONSHIP IN THE XVI CENTURY: SYNTHESIS AND INTERPRETATION IN THE LIGHT OF RECENT HISTORIOGRAPHY}

\author{
Carlos Amate Pizarro ${ }^{1}$ \\ Recibido: 09/09/2021 - Aceptado: 04/10/2021 \\ DOI: https://doi.org/10.5944/etfiv.34.2021.31708
}

\section{Resumen}

El asentamiento de la Monarquía hispánica en Filipinas se produciría tras varias exploraciones fallidas por el Pacífico durante la primera mitad del siglo XVI. Solo la consecución del tornaviaje permitiría el establecimiento de una ruta comercial marítima estable y constante que insertaría a este archipiélago dentro del sistema de la Monarquía. Este hecho haría realidad el sueño de Colón, llegar a Asia a través de una ruta occidental. En este estudio se analiza las investigaciones de los especialistas acerca del establecimiento de los españoles en oriente, su inicio, su desarrollo y cómo se asentaron las bases que permitirían la presencia hispana en esta zona durante más tres siglos.

Palabras clave

Filipinas; Comercio; Asentamiento; China; Monarquía Católica.

\section{Abstract}

The Spanish Monarchy settlement in the Philippines would take place after several fail expeditions along the Pacific during the first half of the sixteenth century. The achievement of the tornaviaje, this is the way back, would allow the establishment of a stable and constant maritime commercial route that will introduce this island in the Spanish Monarchy system. This success would make Colon's dream come true, arrive to Asia taking a western route. In this study it is analyzed the investigations of specialists about the Spanish settlement in Asia, its beginnings, development and how the bases that would allow the Hispanic presence in this place for more than three centuries were established.

1. Universidad Autónoma de Madrid; carlos.amate@estudiante.uam.es 


\section{Keywords}

Philippines; Commerce; Settlement; China; Catholic Monarchy. 


\section{INTRODUCCIÓN}

El establecimiento de una ruta comercial con Asia fue el sueño de Colón, pero no se vería cumplido hasta que Andrés de Urdaneta consiguiera realizar el tornaviaje, es decir, se completara el viaje de vuelta a Nueva España navegando hacia el este. Antes de lograr este objetivo, se realizaron diversas exploraciones por el Pacífico motivadas por conseguir un acceso directo a los productos tan cotizados en Europa en aquellos momentos, como las diversas mercaderías orientales y, especialmente, las especias. En este contexto se encuadra el asentamiento en Filipinas, lugar que se convertiría en punto de encuentro entre los europeos y asiáticos, en particular los chinos hambrientos por plata americana.

Esta plata sería intercambiada por los tan deseados productos orientales, sirviendo para equilibrar la balanza comercial. El resultado, fue la instauración de una ruta comercial continua entre Acapulco y Manila materializada mediante el Galeón de Manila que permitiría la presencia y desarrollo de la Monarquía Católica en aquellas aguas. Esta línea comercial, crearía una relación de dependencia entre ambas comunidades en las que ambas necesitaban productos que los otros podían ofrecer. Este desarrollo económico, además, fue promovido por las políticas de liberalización comercial y el aperturismo hacia el exterior que se empezó a instaurar en los países asiáticos tras la llegada de los ibéricos a la zona en el siglo XVI.

El objetivo de este artículo no es aportar una investigación original al tema, sino retomar algunas de las investigaciones llevadas a cabo por los especialistas y hacer una recapitulación de cómo se concibe la expansión hispana por el Pacífico y su establecimiento en Asia Oriental durante el siglo XVI, con la mirada puesta en el deseado comercio de las especias, el desarrollo del Galeón de Manila, y las aspiraciones españolas en la zona.

\section{EL MITO DE CHINA: DE CRISTÓBAL COLÓN A LEGAZPI, LA RUTA OCCIDENTAL A ASIA}

\subsection{NAVEGACIÓN Y EXPLORACIÓN DEL PACÍFICO: CUMPLIR EL SUEÑO DE COLÓN}

El conocimiento que disponían los europeos de Asia estaba marcado por los relatos, sobre todo de misioneros y comerciantes, de estas tierras orientales. Durante la Edad Media, estos viajeros habían recorrido las rutas hacia Asia amparados en la tolerancia de los Kanes hacia los extranjeros, y la seguridad de las vías terrestres garantizada por la administración mongol. Tras su regreso a Europa, algunos escribían sobre las lejanas tierras visitadas, un ejemplo es el caso del Libro de las Maravillas del comerciante veneciano Marco Polo, redactado hacia I292, que dio a conocer a Occidente las maravillas y riquezas de Catay y Cipango, reinos situados en el extremo más remoto del continente euroasiático. 
Sin embargo, la caída del imperio Mongol y la entronización de la dinastía Ming (I368-i644) provocarían tanto el cierre de las rutas terrestres, como la interrupción de los viajes europeos a Oriente. De hecho, el código Ming, establecido durante el gobierno del emperador Hongwu, prohibía a los ciudadanos chinos la salida del país o la divulgación de información, no obstante, estas restricciones no eran siempre respetadas ${ }^{2}$. Esta ruptura de los caminos y canales de información entre China y Europa explica la persistencia en el ámbito europeo de los escritos de Marco Polo, ya que no se disponía de ninguna otra información relevante acerca de estas tierras.

Esta herencia medieval en la percepción de Oriente provocó que durante el siglo $\mathrm{XVI}$, los ibéricos proyectasen sobre esta área oriental la imagen de la existencia de unas islas asiáticas ricas en metales preciosos ${ }^{3}$. De la misma manera, la búsqueda de prestigio de las clases dirigentes europeas mediante el consumo de especias, sedas orientales y carísimos productos provenientes de Oriente incitó el desarrollo de las exploraciones marítimas a estas zonas. El objetivo de estas navegaciones era establecer rutas comerciales que les diesen acceso directo a estos productos de lujo, lo que daría lugar a una transformación del comercio en este periodo ${ }^{4}$.

En este contexto, Portugal ya había explorado las costas de África, cuando en I488 el lusitano Bartolomé Dias, a través del cabo de Buena Esperanza, consiguió abrir una ruta directa a las sedas y especias orientales. Este hecho, tuvo una notable relevancia en el proyecto de Cristóbal Colón de alcanzar Oriente por una ruta occidental, porque los Reyes Católicos veían como sus rivales en la carrera de la especería conseguían un acceso directo a estos lujosos productos, cuyo valor superaba su peso en oro $^{5}$.

Así pues, la Corona de Castilla apoyaría a Colón en su proyecto. Este partió de Palos en I492, llevando consigo unas cartas diplomáticas para entregárselas al Gran Kan de Catay, y a pesar de que hacía más de una centuria que los mongoles habían sido expulsados de China, el Almirante todavía se guiaba por los legados de la Edad Media ${ }^{6}$. El resultado final, sin embargo, fue totalmente distinto al esperado alcanzando, a lo que posteriormente se denominaría como América, el I2 de octubre de 1492 .

A partir de este momento, surgirían las primeras disputas entre Castilla y Portugal por la extensión de su soberanía sobre los nuevos territorios. El Papa Alejandro VI, mediador entre estos dos católicos reinos, promovió la firma del Tratado de Tordesillas en I493, dividiendo el mundo en dos mitades, una para cada uno de ellos. Esta bula, a pesar de marcar el camino a seguir en la expansión y cristianización, no acabaría con las tensiones fronterizas, debido a su vaga

2. CHAN, (1978): 53-54

3. OLLÉ, (1998b).

4. Sobre las expediciones Ibéricas a extremo Oriente durante el siglo XVI: Barrón Soto, 2012. Bernabeú Albert, 1992. Gil Fernández, 1989. Hidalgo Nuchera, 1995b. Prieto, 2019. Vitorino Magalhaes, 2000. Yun CASALILLA, 2019.

5. GORDON \& MORALES, 2017: 8-10

6. LI, (2018): 102 
definición, al conocimiento incierto de la superficie de la tierra y a los intereses comerciales suscitados por estas nuevas tierras ${ }^{7}$.

Castilla había alcanzado un nuevo continente que acabaría presentando oportunidades difícilmente imaginables para los contemporáneos. Mientras estos se centraban en la conquista de las Indias Occidentales, los portugueses se asentaba en la costa occidental de la India, alcanzaban la península de Malaca o centraban su atención tanto hacia las islas de la especería como hacia a los puertos chinos, donde establecería colonias comerciales, como es el caso de Macao, llegando al Japón alrededor de $1554^{8}$.

En este contexto de exploración tanto castellanos como portugueses tenían un objetivo común, establecer una red comercial directa con las tan deseadas islas de las especias. Prueba de ello fue la expedición dirigida por Fernando de Magallanes y Juan Sebastián Elcano, cuyo resultado daría lugar a la reavivación del conflicto entre estas dos potencias coloniales. Estas tensiones perdurarían en los siglos venideros, solo la aparición de los holandeses en Asia Oriental en siglo XVII provocaría una colaboración entre estos dos competidores, aunque no duraría mucho tiempo ${ }^{9}$.

Esta disputa giraba en torno a la soberanía de estas islas, es decir, si las Molucas se encontraban dentro de la demarcación Portuguesa o Castellana según el Tratado de Tordesillas. En medio de estos debates, España continuó sus exploraciones al Maluco con los mismos objetivos que los anteriores. Así, se preparó una nueva expedición dirigida por García Jofre de Loaísa, y aunque como afirman autores como Peter Gordon y Juan José Morales «fue un tremendo fracaso» ${ }^{\mathrm{ro}}$, la importancia de esta empresa fue la supervivencia de Andrés de Urdaneta que viajaba a bordo de estas embarcaciones. Una última expedición hasta la firma del Tratado de Zaragoza de I529, fue la capitaneada por Álvaro de Saveedra, organizada desde México, y aunque consiguió alcanzar las Molucas, no logró llevar a cabo el viaje de regreso ${ }^{\text {II }}$.

De esta forma, con la firma del Tratado de Zaragoza los españoles hacían un paréntesis en las reclamaciones soberanas de estas islas. En este sentido, Carlos Martínez Shaw ha señalado las razones para el abandono de estas reclamaciones ${ }^{12}$. En primer lugar, los expertos castellanos estaban llegando a las conclusiones de que estas islas se encontraban dentro de la zona expansiva portuguesa. En segundo lugar, Carlos V necesitaba dinero para sufragar las guerras en las que se

\footnotetext{
7. Las tensiones entre ambos países se reabrirán después de la expedición de Magallanes y Elcano, tras completar la primera circunnavegación al globo y volver cargados de clavo de las islas Molucas. Estos litigios se resolverían temporalmente tras la firma del Tratado de Zaragoza en 1529, ya que el interés hispano por la especería, tras la llegada de Legazpi a Filipinas en 1565, reavivaría estas disputas.

8. Siguiendo su modelo de expansión ultramarina, los portugueses establecerían factorías en puntos comerciales estratégicos que actuarían como enclaves mercantiles. Estos emplazamientos locales formarían una red de comercio global que darían forma a la Carrera da India. Para más información véase: SUBRAHMANYAN, (2017): 1359-1385; 2012

9. BOXER, (1946)

10. Gordon \& MORALES, 2017: 10.

11. Prieto, 2019

12. Martínez Shaw, (2019b): 28-29. A este autor se le reconoce internacionalmente por su amplia contribución a entender la presencia hispana en Asia Oriental. Véase: MARTínez SHAW, (2019a): 9-34; (2016): 51-62; (2001): 3-25.
} 
encontraba envuelto en Europa, cosa que aportaba el tratado, porque Portugal tenía que pagar 35.000 ducados de oro para la renuncia española a la soberanía.

En tercer lugar, las relaciones familiares entre los dos reinos no era el contexto más adecuado para seguir alimentado el conflicto. Por último, las experiencias acumuladas por Elcano, Laoísa y Saavedra durante sus expediciones mostraban la dificultad para llevar a cabo la ruta de regreso desde las islas de la especería. Por tanto, en el año I529 se ponía un punto y aparte sobre la apertura de una ruta marítima y comercial directa con las islas Molucas.

\subsection{EL TORNAVIAJE}

En la segunda etapa de las incursiones españolas en el Pacífico, se propuso una alternativa que pasaría por la ocupación de las islas Filipinas y el establecimiento de una ruta que garantizase el contacto regular entre este archipiélago y la costa occidental de la América española. La primera expedición encaminada a conseguir estos objetivos fue emprendida por Ruy López de Villalobos, que tras atravesar las Marshall y las Carolinas desembocó en el archipiélago Filipino. Sin embargo, el asentamiento definitivo en estas islas no se produciría hasta la llegada de la flota dirigida por Miguel López de Legazpi, que fundaría San Miguel, actual Cebú, en I565, y Manila en I57I.

Cortado el acceso directo a las especias, ya aseguradas para Portugal, las Filipinas se convirtieron en la plataforma desde donde acceder a los productos orientales, cuyo suministro se intercambiaría por la plata extraída de América ya que su valor serviría para equilibrar la balanza comercial ${ }^{13}$. Tradicionalmente, se ha considerado que el interés de los castellanos por Asia Oriental se centraba en las islas de la especería y en su fructífero negocio, pero, Patricio Hidalgo sugiere que uno de los objetivos principales era llegar a China, donde la plata americana era ampliamente demandada ${ }^{\mathrm{I}}$.

En los primeros años de la presencia española en el archipiélago, los hispanos se asentaron en Cebú, sin embargo, este no sería el establecimiento final. Así, siguiendo con la teoría de Patricio Hidalgo, si el objetivo eran las Molucas, debían quedarse en Cebú pero, si pretendían llegar a China el asentamiento más acertado era la isla de Luzón. Esta cuestión quedaría esclarecida en la correspondencia escrita en I570 entre Legazpi y el virrey de Nueva España, Martín Enríquez de Almansa:

También querría estar çierto de la voluntad de su magestad si é de cobrar a maluco y lo que más le perteneçe de aquella parte, porque para esto está más cómodo el asiento de çubú que otro por la bondad del puerto, pero si su magestad pretende que sus ministros se estiendan a la parte del norte y costa de china, tengo por más açertadohazer asiento en la ysla de luçón ${ }^{15}$.

13. Para conocer mejor el poder de compra de la plata americana en el sudeste asiático durante los siglos XVI-XVIII: GIRÁLDEZ, 2015; (1995): 201-221. MARTínEZ SHAW, (2015): 21-45. VILLIERS, (1980): 66-80.

14. Hidalgo nuchera, (2009).

15. «Carta de Legazpi al virrey de Nueva España, Panay, 25 de julio de 1570», AGI, Patronato, 24 
Su proceder, nos deja claro que el objetivo era llegar a China, de hecho, Legazpi manda una expedición dirigida por su maestre de campo Martín de Goity, para inspeccionar la isla de Luzón, que acabaría con la fundación de la ciudad de Manila. De la misma manera, Legazpi en otra carta al virrey de Nueva España nos deja aún más claro sus intenciones:

Viniendo el año pasado de panae para este Río, en el camino, en la ysla de vindoro y en otras yslas de su comarca, hallé muchos indios chinos cautivos, que los naturales los tenían por esclavos, (...) y paresçiéndome coyuntura para travar amistad y contrataçión con los chinos, rescaté y compré todos los que se pudieron aver, y les dí libertad para que libremente pudiesen yr a su tierra; fueron treynta y tantas personas las que se libertaron, las qualesembié desde vindoro en vn navío a su tierra; quedaron muy obligados por la buena obra y libertad que se les avía dado ${ }^{16}$.

La opción hacia el imperio Celeste, por tanto, queda reflejada en el derrotero de esta expedición a Filipinas. Esta apuesta hacia la empresa de China, vital para entender la historia de las Filipinas, es reconocida ampliamente por los investigadores ${ }^{17}$.Estas islas se acabarían convirtiendo en una plataforma entre el comercio de Nueva España con este país asiático, sirviendo de puente entre España y Asia Oriental. Legazpi, por su parte, trataría de ganarse la confianza de los chinos con la intención de conseguir información privilegiada sobre su país ${ }^{18}$.

No obstante, y al igual que las anteriores, esta expedición se encontraba con una gran dificultad, cuya resolución marcaría la presencia española en esta zona, esto es, encontrar la ruta de regreso. Para esta fundamental misión, Urdaneta fue el hombre elegido por Felipe II, las instrucciones fueron las siguientes, «no deben retrasarse con ningún rescate o comerciando, deben volver inmediatamente a Nueva España porque el objetivo principal del viaje es aprender la ruta de regreso» ${ }^{19}$.

El navío zarparía de Cebú, navegando en mar abierto ayudado por el monzón de verano. El fin de Urdaneta, era alcanzar los treinta y nueve grados de latitud, donde se encontraba una corriente marina que lo impulsaría rápidamente hacia América. Después de navegar varios días a vista de costa, y tras dejar atrás California, la nao San Pedro avistaba el puerto de Acapulco consiguiendo descubrir el viaje de retorno o tornaviaje ${ }^{20}$. El mayor éxito de esta empresa, fue descubrir esta vuelta de retorno a Nueva España ${ }^{21}$.

Sin embargo, esta expedición transpacífica no es ajena al debate historiográfico acerca de un posible tornaviaje realizado con anterioridad a Urdaneta ${ }^{22}$. En la

16. «Carta de Legazpi al virrey de Nueva España, 11 de agosto de 1572», AGI, Patronato, 24.

17. Así lo afirman autores como: Hidalgo Nuchera, 1995b. Ollé, 2002. NÖEl Sánchez, (2009). Ramón de Miguel, (2008). GIRÁldEZ Y FLYNN, 2015.

18. «Carta de Legazpi al virrey de Nueva España, 11 de agosto de 1572», AGI, Patronato, 24.

19. GORDON \& MORALES, 2017: 15.

20. Algunos de los autores que han considerado el tornaviaje como un éxito colectivo y han restado importancia al papel individual de Urdaneta son: GIL FERNÁNDEZ, (2013). BARANDICA, (2008).

21. Martínez Shaw, (2019b): 29-30.

22. Existen indicios claros de que el San Lucas, embarcación capitaneada por Alfonso de Arellano y que formaba 
actualidad, está bastante aceptado que Alfonso de Arellano atravesó el Pacífico antes de Urdaneta, aunque el verdadero éxito de este agustino reside en la apertura de una ruta transpacífica constante y estable.

Se había cumplido el sueño de Colón, establecer una ruta occidental con Asia. El resultado, la apertura de un comercio regular entre Filipinas y Acapulco, y a la postre, lo que se conocería como una primera globalización ${ }^{23}$. La Monarquía de España, se abastecería desde entonces de especias, porcelanas o sedas provenientes del mundo asiático, mientras exportaba municiones, telas y, sobre todo, metales preciosos.

\subsection{LA EXPLOTACIÓN}

En un primer momento, al igual que los Reyes Católicos intentaron hacer con las Indias, se estipuló la exclusividad comercial de la Corona con el archipiélago excluyendo a todos los agentes privados en la explotación de estas islas. Sin embargo, la continuidad de esta política económica será inviable debido a la falta de metales preciosos y de las especias más demandadas, pimienta y clavo. Como señala AntonioMiguel Bernaldos medias fueron tomadas por parte de la Corona para introducir a las Filipinas dentro del conjunto de la Monarquía ${ }^{24}$. La primera, fue el abandono del usufructo monopolístico estatal y su apertura a sectores privados. La segunda, más debatida y tardía en ejecutar, fue la transmisión del control de la isla de la metrópolis al virreinato de Nueva España.

En realidad, este archipiélago actuaba de forma independiente debido a la gran distancia que lo separaba del resto de territorios. Los contactos entre Madrid y Manila, teniendo en cuenta que las misivas tenían que recorrer un viaje de ida y otro de vuelta, duraban al menos tres años. Así pues, se creó un sistema de gobierno donde el poder estaba en manos de diferentes cuerpos ${ }^{25}$ : la administración de justicia la ejecutaba la Audiencia, un tribunal de cuatro o cinco magistrados, y la administración eclesiástica un arzobispo.

El gobernador, la Audiencia y el arzobispo residían en Manila, acompañados de una guarnición de soldados para la protección de la ciudad. Fuera de la ciudad, los distritos eran dirigidos por gobernadores locales llamados alcaldes mayores y en los lugares alejados de Manila fueron los misioneros los que realizaron toda la labor, la de evangelización e incluso la de gobernantes. Para controlar este sistema burocrático, se recurrían a las visitas que controlaban la correcta actuación de la justicia, la hacienda y la actuación de los funcionarios ${ }^{26}$.

parte de la expedición liderada por Legazpi cuando partieron de América, en noviembre 1564, pudo haber logrado llegar hasta Mindanao y haber regresado a México el 9 de agosto de 1565, dos meses antes que lo hiciera Urdaneta.

23. En la actualidad, la historiografía paulatinamente está poniendo en relieve el papel fundamental de los pueblos ibéricos en la primea globalización, globalización temprana o globalización ibérica. Véase, por ejemplo: Gruzinski, 2004. Martínez Torres, 2014. Giráldez, 2015. Hausberger, 2018.

24. Bernal Rodríguez, 2004: 448.

25. García-Abásolo González, (2013): 18-19. Para más información sobre el funcionamiento de la Audiencia de Manila, véase: Hidalgo Nuchera, 2012.

26. Hidalgo Nuchera, (2001): 207. 
Este sistema administrativo, al inicio de la presencia española en Filipinas, era financiado por el socorro novohispano. Sin embargo, la Corona pretendía crear un estímulo económico que atrajera a nuevos pobladores, con el objetivo de mantener un contingente militar sin la necesidad del socorro, y así asegurar la presencia castellana en Asia ante la insuficiencia de las encomiendas ${ }^{27}$. Sin embargo, las Filipinas eran incapaces de producir el excedente que los españoles ansiaban, y todavía las relaciones con China se encontraban en fase de desarrollo.

En esta situación, el conflicto entre los religiosos y los encomenderos acerca del trato a los nativos ${ }^{28}$ agudizaría las tensiones entre los pobladores de estas islas lo que daría lugar a un incremento en las voluntades alrededor de un posible proceso expansivo hacia China $^{29}$. Se reactivaría, por tanto, una polémica acerca de las bases en las que se debía asentarse el dominio español en estas posesiones asiáticas.

Por un lado, estaba la postura sostenida por el Consejo de Indias y compartida por el gobernador Gómez Pérez Dasmariñas que proponían mantener la presencia española mediante el comercio de los productos de este archipiélago en México. Por otro lado, estaría el modelo de intermediación que pasaría por ampliar el comercio con China y permitir el paso suficiente de plata mexicana para estimular la continuidad española ${ }^{30}$. Su adopción, en ambos casos, se conseguiría mediante el establecimiento de una ruta comercial entre Manila y Acapulco ${ }^{31}$.

Finalmente, la primera opción tuvo que ser descartada debido al fracaso de las riquezas naturales en la obtención de excedente, optándose por el intercambio de mercaderías orientales (sedas, porcelanas etc..) por plata mexicana. De hecho, la mayoría de la plata acabaría en manos de los chinos que la enviarían a su tierra natal, donde obtendrían unas importantes ganancias al duplicarse su valor ${ }^{32}$. Por tanto, la economía Filipina se basaría en el comercio y en el alquiler de las tiendas del mercado, convirtiéndose estas actividades en las más lucrativas del archipiélago. Estas dos actividades, permitirían el asentamiento, desarrollo y permanencia de la Monarquía Católica en las islas ${ }^{33}$.

27. Ibíd, 1995a.

28. Se producirían tensiones entre encomenderos y religiosos acerca del trato a los indígenas y a la explotación de aquellas nuevas tierras. Esto se debió al cambio de mentalidad acerca de la legitimación jurídico-teológica de la presencia española en Filipinas. Esta doctrina de dominio promulgada por Bartolomé de las Casas y Francisco de Vitoria tras la ocupación de América, difiere del discurso oficial y marco legal de la conquista americana, basando el trato a los indígenas en el derecho internacional de gentes. Para más información, véase: OLLÉ, 1999: 94-102.

29. MANEL OlLÉ ha tratado ampliamente la hipótesis acerca del interés de los españoles por entablar relaciones con China, primero mediante el establecimiento de un enclave comercial similar al de Macao, y luego a través del Galeón de Manila. Entre sus numerosas obras pueden citarse las siguientes: OLLÉ, 2002; (2013a): 155-178; (2013b): 253-277; (2014): 371-390.

30. Alonso Álvarez, (2001): 182-183.

31. La apertura de esta ruta comercial marítima generaría una serie de conflictos y arbitrismos en el interior de la Monarquía, debido a que los comerciantes sevillanos sentirían atacados sus intereses comerciales en América por los productos asiáticos, de menor precio y de gran calidad. Estas disputas quedarían formalmente dirimidas con el Reglamento de 1593. Alonso Álvarez, (2013): 25-84.

32. Se produciría el ocaso del comercio de las especias, y el alba de la plata y la seda como mercaderías que dinamizarían y desarrollarían el tráfico comercial en Asia Oriental. Estas mercaderías permitirían el nacimiento de un comercio global que se pude fechar en 1571, año de la fundación de la ciudad de Manila. Así lo afirman: FLYNN \& GIRÁLDEZ, (1995): 201.

33. Varios autores han demostrado que las Filipinas eran una fuente de ingresos y una posesión económicamente viable dentro del Imperio y no una propuesta fracasada, ya que los tributos de las islas y los derechos del Galeón 
A este respecto, Manel Ollé ha señalado dos factores principales que motivaron el desarrollo de este comercio marítimo ${ }^{34}$. El primero, el fenómeno de monetarización de la economía China que a la llegada de los españoles estaba en pleno auge. La plata se convirtió en el principal producto demandando en el intercambio con el exterior generándose un flujo comercial hacía Asia canalizado a través del Galeón de Manila. El segundo, la liberalización del comercio marítimo por parte del gigante

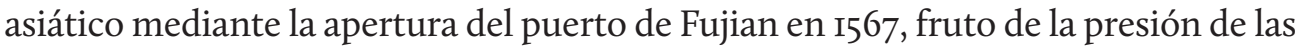
élites locales y de la disminución de la piratería japonesa.

Este modelo económico basado en el comercio, fue integrando progresivamente los mercados del Pacífico y del Atlántico ${ }^{35}$. Las directrices de esta actividad comercial quedarían fijadas en el Reglamento de I593, el cual regulaba las transacciones mercantiles de Nueva España con China a través del Galeón ${ }^{36}$. Este barco no solo designaría a un instrumento lucrativo sino también a una ruta comercial que permitiría la conexión de los diferentes mercados locales y la interacción sociocultural de los europeos con sus coetáneos asiáticos.

Esta línea de intercambios constaría de un barco anual que partiría desde Cavite, puerto cercano a Manila, en el mes de julio llegando a Acapulco en el mes de diciembre, donde se realizaría una feria anual con la participación de comerciantes de diferentes partes de México. Su partida hacia el archipiélago filipino se producía, por lo general, en marzo para que su descarga estuviese lista en julio. Así pues, como señala Carlos Martínez Shaw los galeones partían desde Filipinas cargados de productos orientales (sedas y porcelanas principalmente), aunque también con objetos japoneses, muebles, marfiles de la India portuguesa, especias (pimienta, clavo o canela) o productos filipinos ${ }^{37}$. En el viaje de retorno, se cargaban remesas de plata (entre un $96 \%$ y un $99 \%$ del total), en su mayoría mexicana, aunque también podía haber plata peruana, complementado con otros productos como la grana, el jabón y los envíos oficiales (papel sellado o artículos destinados a los Reales Almacenes) ${ }^{38}$.

De esta manera, se impondría el suministro de productos asiáticos dentro de este modelo económico que aseguraba el mantenimiento sin que supusiera unos gastos para la Corona. La importancia de la fiscalidad de las encomiendas se vería ensombrecida hasta convertirse en un elemento residual en la economía de Filipinas ${ }^{39}$. Los encomenderos, aceptando la nueva realidad se convirtieron en cargadores del Galeón.

de Manila permitían equilibrar los gastos que la Corona tenía en esta posesión ultramarina. Aunque, el traslado y recrudecimiento del conflicto holandés en estos territorios, obligaría a una mayor dependencia económica de México. VAldés Lakowsky, (1987): 84. Flynn \& Giráldez, (1999): 32. Alonso ÁlvareZ, (2009): 267-268. GIL, $2011: 146$.

34. OLLÉ, (2001): 63-64.

35. ElLiot, 2002.

36. Esta Ordenación de 1593, aprendida a base de aciertos y errores, regulaba el comercio entre Filipinas y Nueva España a favor de los vecinos de Manila fijando su prioridad sobre la carga de mercaderías en el Galeón de Manila y el exclusivo comercio de estas islas con México. Además, se restringía el tráfico directo con el continente asiático, teniendo que ser las mercaderías dirigidas a Manila. Se establecía, asimismo, dos galeones anuales financiados, a diferencia de la carrera del Atlántico, con capital público proveniente de los almojarifazgos y fletes. Por último, se exigía la visita y registro del gobernador en Manila y el virrey en Acapulco. Sin embargo, y a pesar de las quejas, está ordenación no se cumpliría estrictamente. Alonso Álvarez, (2013): 69-71, 80.

37. Martínez ShaW, (2019b): 30

38. Ibíd, (2019b): 31.

39. Alonso Álvarez, (2001): 203-204. 


\section{LA PROYECCIÓN ESPAÑOLA EN ASIA: EL MAR DE LA CHINA HISPÁNICO}

El entorno regional que rodeaba a las islas Filipinas durante las primeras décadas de dominación hispánica moldeó el sistema colonial desarrollado en esta isla y su proyección exterior. Este entorno se ha definido bajo el concepto del mediterráneo del mar de la China ${ }^{40}$. Tras los primeros años, los españoles se dieron cuenta de que la economía filipina era una economía de autoconsumo y no podrían explotarla como deseaban, no tenía la plata de Perú ni las especias de las islas Molucas. Además, la resistencia de los originales de las islas y las polémicas con la iglesia hacían que los encomenderos se mostrasen reacios a establecerse en aquel archipiélago.

En este marco de escaso beneficio comercial y fiscal, junto con la lejanía de Filipinas de la metrópoli, se potencia el intercambio comercial con agentes exteriores como único camino para el mantenimiento de un contingente español en las islas. En la ciudad de Manila, tendría lugar un encuentro multicultural entre los diferentes comerciantes que se acercaban a este archipiélago a intercambiar sus productos. Estos intercambios se convirtieron en el elemento esencial para el mantenimiento de este contingente, contribuyendo a la formación del sistema colonial filipino y al progresivo control insular.

Antes de la llegada de los españoles a Filipinas, existía un importante comercio en este mediterráneo del mar de la China. En este archipiélago, encontramos testimonios de comercio con las Molucas realizado a través de las rutas comerciales de Borneo y el mar de Sulú, o los vínculos comerciales nipo-filipinos y sino-filipinos presentes en los sultanatos musulmanes de la zona $^{41}$. Tras su llegada a Filipinas, los españoles se dieron cuenta de que Manila era un puerto de escala y permanencia de mercaderes chinos, cuya importancia a nivel de mercaderías y volumen de intercambio crecería tras el asentamiento español en Manila ${ }^{42}$.

$\mathrm{Al}$ igual que hicieran en América, los españoles llevarían a cabo proyectos de expansión y dominio militar de la zona siguiendo su modelo de conquista para el control de los territorios. Así había acontecido también en la conquista de las islas Canarias y en las campañas del norte de África a principios del siglo XVI. El objetivo, la consecución de privilegios por parte de las oligarquías y la satisfacción de las necesidades por parte de la Corona, nobleza, Iglesia y ciudades que aliviaban de esta manera el conflicto entre ellas. En última instancia, se pretendía ampliar el Imperio, extender la cristianización y obtener ventajas comerciales ${ }^{43}$.

De esta manera, una vez asegurada Manila y después de advertir la inexistencia de abundantes especias o metales preciosos, se elaboraron planes de conquista

\footnotetext{
40. OlLÉ, (2001): 59 .

41. PTACK, (1992): 38.

42. La llegada de los ibéricos a esta zona, primero los portugueses a Malaca y luego los españoles a Filipinas, coincidió con la relajación de la política de haijin. Esta política se caracterizó por un feroz aislamiento con el exterior propio de la dinastía Ming, y en contraposición con un periodo más belicista y de un mayor intervencionismo político-militar de la dinastía Yuan, especialmente en Vietnam y Java. Véase: De SouSA PINTO, (2013): 91-109.

43. Para conocer más acerca de las bases sobre las que se asentaba la dinámica expansionista de la Monarquía Hispánica, véase: Rivero Rodríguez, 2016: 305. Yun CaSALILla, 2019: 17-18, 29-31.
} 
de Borneo, Mindanao y otras islas del Pacífico. Es posible, como sugiere Alicia Castellanos Escudier, que en estas fechas aún estuviera en el recuerdo la victoria de Lepanto (157I) que tanto prestigio y seguridad había otorgado a los españoles ${ }^{44}$. El primer dirigente del gobierno de Filipinas que organizó una expedición militar destinada a controlar y someter al sultán de Borneo fue Francisco de Sande en I578. En un primer momento, se apuesta por una vía pacífica enviándose una embajada para que el sultán se convirtiese en vasallo del rey, se reanudaran las transacciones comerciales y se permitiese la entrada de misioneros españoles.

Sin embargo, la negatividad mostrada a los enviados españoles conducirá a un resultado inevitable, el conflicto militar. Tras varios años de disputas, el acuerdo formal con el reino de Borneo llega durante el gobierno de Francisco Tello de Guzmán en 1599. El resultado, el aumento de la presencia española en el norte de Borneo, lo que supuso una serie de beneficios para su consolidación en la zona. Primero, por la sumisión de los dirigentes musulmanes de Filipinas, que suponían una amenaza para los castellanos en caso de una alianza contra ellos. Lo segundo, por la situación estratégica tan importante para el desarrollo comercial de las islas. Sin embargo, los españoles no conseguirán todas las reclamaciones que demandaban, negándose la entrada de misioneros a la isla ${ }^{45}$.

A la vez que se llevaba a cabo una política activa sobre Borneo, se sometieron las sultanías de Mindanao y Joló aliadas de este sultanato. Al igual que hiciera Sande, Ronquillo aprovecha una campaña militar sobre la isla de Borneo para mandar la armada con embajadas a los reinos de Patán, Siam y Camboya con la intención de llegar a acuerdos comerciales. De la misma manera, en los últimos decenios del siglo XVI, el punto de mira se centraría en la isla nipona. Como sugiere Juan Gil Fernández el fin de los ideales caballerescos y el surgimiento de una nueva mentalidad suntuaria, fue un acontecimiento determinante en las relaciones con la isla ya que obligó a Japón a permitir la entrada de mercancías que permitiesen hacer gala de la ostentación de riqueza ${ }^{46}$.

Estos intereses mercantiles y los asaltos piráticos eran los puntos de encuentro entre Manila y Japón, sin embargo, la posición de Portugal, asentados ya firmemente en el puerto comercial de Nagasaki, suponía un obstáculo importante, especialmente tras su incorporación a la Monarquía en i58I. Los portugueses, habían aprovechado la rivalidad y enemistad sino-japonesa para convertirse en los intermediaros comerciales entre ambos países. Cargarían la plata demanda en China en el puerto de Nagasaki donde la intercambiarían por las sedas de alta calidad chinas. Así, una hipotética introducción de los españoles en el mercado nipon, disponibles de una amplia oferta de plata americana, causo un amplio receló entre los portugueses que intentarían obstaculizar su entrada en este comercio. Por tanto, y al igual que ocurriera en China, las relaciones de los ibéricos en Japón

\footnotetext{
44. Castellanos Escudier, (2016): 22

45. Castellanos Escudier, (2016): 23.

46. FERNÁNDEZ GIL, 1991: 33.
} 
variarían entre la rivalidad y la colaboración según los intereses, las circunstancias y las necesidades del momento ${ }^{47}$.

En esta dinámica, la Unión Ibérica amplió el horizonte de ambos Imperios al entrelazarse proyectos e intereses en Asia Oriental. Sin embargo, y a pesar de prometerse la instauración de un régimen político que respetara la exclusividad de los dos Imperios en sus respectivas posesiones, ambos intentarán, directa o indirectamente, obtener beneficios de la Unión a la vez que intentan monopolizar rutas comerciales y misiones espirituales. Los castellanos, intentarían expandirse hacia áreas vinculadas al portugués asiático, multiplicándose las acciones para controlar el comercio de las especias o introducirse en el puerto comercial de Macao ${ }^{48}$. La consecuencia de estas interacciones, sería un comercio informal constante entre ambos países motivado por la lejanía de estos lugares de los centros de decisión de la Monarquía.

Por otro lado, las primeras décadas de las relaciones nipo-españolas estuvieron marcadas por una política belicosa y un clima de tensión ante un posible ataque por cualquiera de las dos partes. Aunque, en este sentido, los españoles se mostraron más cautelosos al encontrarse en una situación más comprometida al depender del socorro novohispano en caso de conflicto armado. Así, se enviarán embajadas por ambas partes con la intención de persuadir al otro ante cualquier posible tentativa de invasión: la delegación liderada por Juan Cobo, llevó un globo terráqueo donde mostró al soberano japonés, Toyotomi Hideyoshi nombrado en las fuentes españolas como Taicosoma, los reinos bajo dominio de Felipe II, señalando la distancia entre uno y otro para que viera el poderío del soberano español. Por su parte, Hideyoshi mostró al enviado desde Manila la capacidad militar y de resistencia que tenían sus dominios ${ }^{49}$.

A la vez que se producía este intercambio diplomático, tuvo lugar un comercio entre Filipinas y Japón que utilizó estas embajadas como pretexto. La muerte de Taicosoma provocó una serie de cambios marcados por la aplicación de una política de acercamiento a los españoles. A partir de 1602 , la correspondencia entre ambos países se convirtió en normalidad, estableciéndose una relación fija por medio de un barco anual que conectaba ambos puertos. Sin embargo, el establecimiento de un puerto comercial holandés en I609, junto con los intentos de expulsar a los misioneros provocará la degeneración de estas relaciones ${ }^{50}$. De la misma manera, como sugiere Fang Chen, la conquista de Taiwán respondería al intento de los españoles de acercarse de manera directa tanto a estos mercados japoneses como chinos, así como una defensa contra los holandeses presentes en estas aguas desde comienzos del siglo XVII ${ }^{51}$.

47. OLLÉ, (2014): 371-390.

48. Estas relaciones, como se ha comentado, no fueron estáticas sino que oscilarían según la circunstancia del momento, y solo la entrada de un tercer sujeto, los holandeses, conseguirá la colaboración entre ambos países motivada más por la necesidad, al encontrarse aquellas posesiones asiáticas en la periferia de la periferia, que por voluntad propia. Para saber más sobre esta relaciones, véase: BOXER, (1946): 150-164; (1985): 51-59.

49. FernándeZ GIL, 1991: 34-35

50. TREMML-WeRner, 2015: 209-238, 265-291

51. Cheng, (2016): 53-74. 
Por último, la proyección española en el mediterráneo del mar de la China estuvo marcada y entrelazada por las relaciones hispano-chinas ya que sin los sangleyes ${ }^{52}$ de Manila, no se puede entender el devenir de la presencia castellana en Asia. El primer paso hacia el mundo chino había sido el asentamiento en Filipinas de la misma manera que las Antillas lo habían sido en el continente americano. Desde la llegada de los españoles a esta parte del mundo, se intentaron establecer relaciones con China, primero por medio de embajadas, y después vía conquista militar, con unos proyectos de conquista tan optimistas como irreales. Sin embargo, las relaciones se trasladarían finalmente del continente asiático a Manila, donde tuvo lugar un encuentro multicultural entre dos mundos diferentes y que participó en la formación del sistema colonial español en oriente y en el desarrollo del sistema del Galeón de Manila, fuente principal de ingresos de los españoles en $\mathrm{Asia}^{53}$.

\section{CONCLUSIONES}

Las exploraciones hacia Asia tenían como objetivo establecer una ruta comercial que permitiera el acceso directo a los productos de lujo asiáticos tan deseados por los europeos. En este contexto, las especias se convirtieron en el motor fundamental de estas exploraciones por el Pacífico desembocando en importantes tensiones entre portugueses y españoles por el dominio comercial de este producto. Estas disputas se cerrarían tras la firma del Tratado de Zaragoza que ponía fin a esta primera etapa de navegaciones por el Pacífico, aunque se volverían a reabrir tras la llegada de Legazpi a la zona. Solo la aparición de un rival común, los holandeses, y dentro de la Unión Ibérica llevaría a una colaboración de ambos países en aquella zona.

Cerrado el acceso a las especias, los castellanos continuaron en su empeño por establecer un comercio directo con China, de hecho, el objetivo de llegar a este lugar fue compartido tanto por Colón como Legazpi. De esta manera, la ocupación de Filipinas y la fundación de Manila responden a estos intereses por acercarse al país asiático. Este asentamiento coincide con la apertura al comercio exterior marítimo en China, al relajarse la política de haijin, y con el cambio de su sistema fiscal caracterizado por la monetarización de la economía lo que motivó la llegada de chinos provenientes, en particular, de la provincia de Fujian.

Filipinas se convertiría en un lugar de encuentro donde comprar y vender productos orientales (especialmente seda y porcelana) por plata americana, producto que serviría para equilibrar la balanza comercial. Así, se crearía una relación de dependencia entre chinos y españoles en la que ambos necesitaban los productos que los otros ofertaban. Los sangleyes, acabarían por dominar la vida económica de Filipinas, controlando el comercio del Galeón al ser los principales abastecedores

52. Los chinos en Manila, provenientes de la provincia de Fujian, aparecen designados por las fuentes españolas como sangleyes, término utilizado para designar a la gente que viene y va, refiriéndose al comercio regular entre China y Manila. La presencia de los sangleyes en Filipinas ha sido analizada por: Fernández GiL, 2011. GarcíaAbásolo GonZÁlez, (2013): 18-19 y el ya citado MANel Ollé.

53. OLLÉ, 1998a. 
de los productos, haciéndose imprescindibles para la continuidad de la presencia española en Asia. Este archipiélago, y a pesar de los intentos españoles de establecerse en las costas chinas, acabaría desempeñando unas funciones similares al enclave comercial luso de Macao.

De la misma forma, los españoles tratarían de entrar en otros mercados asiáticos, como el japonés, mediante el envío de embajadas para establecer un enclave mercantil, al igual que el puerto portugués de Nagasaki. Por otro lado, también ampliarían su presencia en la zona mediante acciones bélicas orientadas a la conquista para el control de los territorios, con el objetivo de asegurarse el acceso a las mercaderías orientales, y defenderse de sus rivales europeos presentes en aquella zona desde comienzos del siglo XVII. Estas acciones armadas demuestran que seguiría habiendo una dinámica imperial al ser la conquista la razón de ser de la Monarquía hasta la paz de Westfalia.

En definitiva, españoles y asiáticos se acabarían encontrando en la ciudad de Manila creándose el primer comercio internacional, al unirse los diversos continentes por medio del Galeón de Manila. Por tanto, los españoles conseguirían el objetivo planteado por Colón, establecer un vínculo con China, mantenimiento su presencia en Asia oriental por más de trescientos años, convirtiéndose Filipinas en el enclave asiático del Imperio español. 


\section{BIBLIOGRAFÍA}

Alonso Álvarez, Luis, «La inviabilidad de la hacienda asiática. Coacción y mercado en la formación del modelo colonias en las Islas Filipinas I565-I595», en María Dolores Elizalde Pérez-Grueso, Josep María Fradera \& Luis Alonso Álvarez (eds), Imperios y naciones en el Pacífico, Madrid, Consejo Superior de Investigaciones Científicas, 200I: I8I-205.

Alonso Álvarez, Luis, El costo del Imperio Asiático: la formación colonial de las islas Filipinas bajo dominio español 1565-I800, México, Instituto Mora, 2009.

BARANDICA, Luis Abraham, «Andrés de Urdaneta en la Nueva España (I538-I568)», en Cristina Barrón (ed.), Urdaneta novohispano. La inserción del mundo hispano en Asia, México, Universidad Iberoamericana, 20I2: 35-65.

Barrón Soto, Cristina, Urdaneta novohispano. La inversión del mundo hispano en Asia, México, Universidad Iberoamericana, 2012.

Bernabeú Albert, Salvador, El Pacífico Ilustrado: Del lago español a las grandes expediciones, Madrid, Mapfre, I992.

Bernal Rodríguez, Antonio Miguel, «La Carrera del Pacífico. Filipinas en el sistema colonial de la carrera de Indias», en Leoncio Cabrero Fernández (ed), España y el Pacífico: Legazpi, España, Sociedad Estatal de Conmemoraciones Culturales, 2004: 485-526.

Boxer, Charles, «Portuguese and Spanish Rivalry in the Far East during the $17^{\text {th }}$ century», Journal of The Royal Asiatic Society of Great Britain and Ireland, 2/I (I946): I50-I64.

Boxer, Charles, «A note on the triangular trade between Macao, Manila and Nagasaki, I580-I640», Taylor and Francis Online, I7/I (I985): 5I-59.

Castellanos Escudier, Alicia, «Expediciones españolas a Borneo en el siglo XVI», en Salvador Bernabéu Albert, Carmen Mena García \& Emilio José Luque Azcona (eds.), Filipinas y el Pacífico. Nuevas miradas, nuevas reflexiones, Sevilla, Universidad de Sevilla, 20I6: 2I-5I.

CHen, Fang, «Colonias españolas en Asia en el siglo XVII: relaciones entre Manila y San Salvador (norte de Taiwán)», en Salvador Bernabéu Albert, Carmen Mena García \& Emilio José Luque Azcona (eds.), Filipinas y el Pacífico. Nuevas miradas, nuevas reflexiones, Sevilla, Universidad de Sevilla, 2016: 53-74.

De Sousa Pinto, Paulo Jorge, «Malaca, Manila e Batávia. Os chineses ultramarinos no contexto dos impérios europeus na Ásia do Sueste (séculos XVI-XVII)», en Manuel Lobato \& Maria de Deus Manso (eds.), Mestiçagens e Identidades. Intercontinentais nos espaços lusófonos, Braga, Núcleo de investigaçao em ciencias políticas e relaçoes internacionais, 2013: 9I-IO9.

Elliot, John, Imperial Spain: I469-I7ı6, New York, Penguin Books, 2002.

FLYNN, Dennis \& GiRÁLDEZ, Arturo, «Born with a silver spoon», Journal of World History, 6/2 (I995): 2OI-22I.

FLYNN Dennis \& GiRÁldez Arturo, «Spanish profitability in the Pacific: The Philippines in the sixteenth and seventeenth century», en Dennis Flynn, Lionel Frost \& J.H. Latham (eds.), Pacific Centuries: Pacific and Rim Histories Since the Sixteenth Century, New York, Routledge Press, I999: 23-37.

García-Abásolo González, Antonio Francisco, Murallas de piedra y cañones de seda. Chinos en el Imperio Español (siglos XVI-XVIII), Córdoba, Universidad de Córdoba, 2012.

García-Abásolo GonzÁlez, Antonio Francisco, «Españoles y chinos en Filipinas, los fundamentos del comercio del Galeón de Manila», en Felipe Lorenzana de la Puente 
(ed.), España, el Atlántico y el Pacífico y otros estudios sobre Extremadura, Llera, Sociedad Extremeña de historia, 2013: 9-30.

Gil Fernández, Juan, Mitos y Utopías del Descubrimiento, vol. 2: El Pacífico, Madrid, Alianza Editorial, I989.

GIL FERNÁNDEZ, Juan, Hidalgos y samuráis España y Japón en los siglos XVI y XVII, Madrid, Alianza Editorial, I991.

Gil Fernández, Juan, Los chinos de Manila (siglos XVI-XVII), Lisboa, Centro Científico e Cultural de Macau, 20II.

Gil FernándeZ, Juan, «El primer tornaviaje», en Salvador Bernabéu Albert (ed.), La nao de China, 1565-I815. Navegación, comercio e intercambios culturales, Sevilla, Universidad de Sevilla, 2013: 25-64.

GiRÁLDEZ, Arturo, The age of Trade. The Manila Galleons and the Dawn of the Global Economy, Londres, Rowman \& Littlefield, 2015.

Gordon, Peter \& Morales, Juan José, The silver way. China, Spanish America and the birth of globalization, 1565-I8I5, Australia, Penguin Group, 2017.

GruZINSKI, Serge, «Les Quatreparties du Monde. Histoire d'une mondialisation», Critique internationale, 26/I (2005): 166-I70.

Hausberger, Bernd, Historia mínima de la globalización temprana, México, Colegio de México, 2018.

Hidalgo Nuchera, Patricio, Encomienda, Tributo y Trabajo en Filipinas (I570-I608), Madrid, Miraguano y Polifemo, I995a.

Hidalgo Nuchera, Patricio, Los primeros de Filipinas. Crónicas de la conquista del archipiélago, Madrid, Miraguano y Polifemo, 1995b.

Hidalgo Nuchera, Patricio, «Visitas a la tierra durante los primeros tiempos de la colonización de las Filipinas, 1565-I608», en María Dolores Elizalde Pérez-Grueso, Josep Maria Fradera \& Luis Alonso Álvarez (eds), Imperios y naciones en el Pacífico, Madrid, Consejo Superior de Investigaciones Científicas, 200I: 207-225.

Hidalgo Nuchera, Patricio, «La figura de Andrés de Urdaneta en la historiografía indiana, conventual, documental y moderna», en Susana Trechuelo García (ed), Andrés de Urdaneta: un hombre moderno, Ordizia, Ayuntamiento de Ordizia, 2009: 17-9I.

Hidalgo Nuchera, Patricio, Los autos acordados de la Real Audiencia de las islas Filipinas de 1598-1599, Madrid, Universidad Autónoma Ediciones, 2012.

LI, Chenguang, La estrategia China de Felipe II: La vía castellana (1556-158I), Madrid (Tesis Doctoral), Universidad Autónoma de Madrid, 2018.

Martínez SHAw, Carlos, «La exploración española del Pacífico en los tiempos modernos», en María Dolores Elizalde Pérez-Grueso, Josep María Fradera, Luis Alonso Álvarez(eds), Imperios y naciones en el Pacífico, Madrid, Consejo Superior de Investigaciones Científicas, 200I: 3-25.

Martínez Shaw, Carlos \& Bernabéu Albert, Salvador, Un océano de seda y plata, el universo económico del Galeón de Manila, Sevilla, Consejo Superior de Investigaciones Científicas, 2013.

MARTínez Shaw, Carlos, «La plata española, catalizador de la primera globalización», en Comercio y cultura en la Edad Moderna: Actas de la XIII reunión científica de la fundación española de historia moderna, Sevilla, Universidad de Sevilla, 2015: 2I-45.

Martínez Shaw, Carlos, «El Galeón de Manila y la economía filipina (I565-1815)», Boletín económico de ICE, Información Comercial Española, 3074/I (2016): 5I-62.

Martínez Shaw, Carlos, «El Galeón de Manila: 250 años de intercambios», Estudis: Revista de historia moderna, 45/I (2019a): 9-34. 
Martínez Shaw, Carlos, «La primera globalización», Andalucía en la historia, 63/2 (2019b): 28-33.

MARTíNEZ ToRres, José Antonio, «There is but one world: Globalization and connections in the overseas territories of the Spanish Habsburgs (158I-I640)», Culture and History Digital Journal, 3/I (2014): I-I5.

Nöel SÁNChez, Jean, «Tiempos Malucos. España y sus Islas de las Especias, I565-I663», en Susana Truchuelo García (ed). Andrés de Urdaneta: un hombre moderno, Ordizia, Ayuntamiento de Ordizia, 2009: 62I-650.

Ollé, Manel, Estrategias Filipinas respecto a China: Alonso Sánchez y Domingo de Salazar en la empresa de China (158I-1593). Barcelona (Tesis Doctoral), Universidad Pompeu Fabra, I998a.

OlLÉ, Manel, «La invención de China: Mitos y escenarios de la imagen ibérica de china en el siglo XVI», Revista Española del Pacífico, 8/I (1998b):360-380.

OlLÉ, Manel, «La invención de China. Percepciones y estrategias filipinas respecto a China durante el siglo XVI», Wiesbaden, Harrossowitz, 2000.

OlLÉ, Manel, «El mediterráneo del mar de la China: dinámicas históricas de Asia oriental y la formación del modelo colonial filipino», en María Dolores Elizalde Pérez-Grueso, Josep María Fradera, Luis Alonso Álvarez (eds), Imperios y naciones en el Pacífico, Madrid, Consejo Superior de Investigaciones Científicas, 2001: 27-38.

Ollé, Manel, La empresa de China. De la Armada Invencible al Galeón de Manila, Barcelona, Acantilado, 2002.

OlLÉ, Manel, «La proyección de Fujien en Manila: los sangleyes del Parián y el comercio de la Nao de China», en Salvador Bernabéu Albert \& Carlos Martínez Shaw (eds.), Un océano de seda y plata: el universo económico del Galeón de Manila, Sevilla, Consejo Superior de Investigaciones Científicas, 2013a: 155-I78.

OlLÉ, Manel, «Portugueses y Castellanos en Asia Oriental», en Pedro Cardim, Leonor Freire Costa \& Mafalda Soares da Cunha (eds.), Portugal na monarquía hispánica. Dinámicas de integraçao e de conflicto, Lisboa, Universidad dos Açores, 2013b: 277-309.

OlLÉ, Manel, «Entre China y la Especería. Castellanos y portugueses en Asia Oriental», en Carlos Martínez Shaw \& José Antonio Martínez Torres (eds.), España y Portugal en el mundo (I58I-I668), Madrid, Ediciones Polifemo, 2014: 37I-390.

Prieto, Carlos, El océano Pacífico. Navegantes españoles del siglo XVI, Madrid, Alianza editorial, 2019.

PTAK, Roderich, «The Northern Trade Route to the Spice Islands: South China Sea - Sulu Zone - North Moluccas (I $4^{\text {th }}$ to early I6 $6^{\text {th }}$ century)», Archipel, 43/I (I992): 27-56.

Ramón de Miguel, José, Urdaneta y su tiempo, Ordizia, Ayuntamiento de Ordizia, 2008.

Rivero Rodríguez, Manuel, La monarquía de los Austrias: Historia del Imperio español, Madrid, Alianza Editorial, 2016.

Subrahmanyam, Sanjay, «Holding the World in Balance: The connected histories of the iberian overseas empires, I500-I640", The American Historial Review, II2/5 (2007): 1359-1385.

Subrahmanyam, Sanjay, The portuguese Empire in Asia 1500-I700, Chichester, Wiley Blackwell, 2012.

Tremml-Werner, Birgit, Spain, China and Japan in Manila, I57I-I644, Amsterdam, Amsterdam Univertiy Press, 2015.

VALDÉS LAKOWSKY, Vera, De las minas del mar: Historia de la plata mexicana en Asia, I565-I834, México, Fondo de cultura económica, 1987.

VILLIERS, John, «Silk and Silver: Macau, Manila and Trade in the China sea in the sixteenth century», Royal Asiatic Society Hong kong Branch, 20/I (1980): 66-80. 
Vitorino Magalhaes, Godinho, Os descubrimientos e a economia mundial, volumen III, Lisboa, Editorial Prensça, 2000.

YUN CASALILLA, Bartolomé, Los imperios ibéricos y la globalización de Europa (siglos XVI-XVII), Barcelona, Galaxia Gutenberg, 2019.

\section{FUENTES PRIMARIAS}

«Carta de Legazpi al virrey de Nueva España, Panay, 25 de julio de I570», AGI, Patronato, 24. «Carta de Legazpi al virrey de Nueva España, II de agosto de I572», AGI, Patronato, 24. 



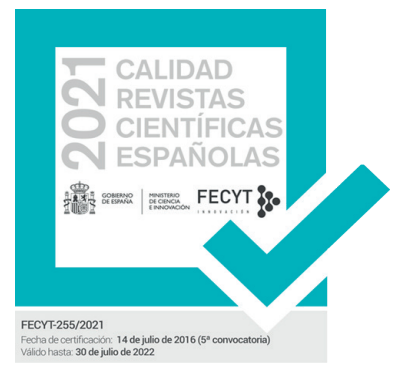

SERIE IV HISTORIA MODERNA

REVISTA DE LA FACULTAD DE GEOGRAFÍA E HISTORIA

AÑO 2021

ISSN: 1131-768X

E-ISSN 2340-1400

\section{4 \\ 西 ESPACIO, TIEMPO Y FORMA}

Monográfico - Special Issue: La política ultramarina de las monarquías ibéricas (circa 1700-1750): una historia de fracasos y éxitos relativos The Overseas Policy of the Iberian Monarchies (Circa 1700-1750): A History of Failures and Relative Successes

15 Roberto Quirós Rosado y MARIA FERnANDA BICALHO La política ultramarina de las monarquías ibéricas (circa 1700-1750): una historia de fracasos y éxitos relativos / The Overseas Policy of the Iberian Monarchies (Circa 1700-1750): A History of Failures and Relative Successes

\section{Guillaume Hanotin}

Defender negocios en tiempo de convulsión política: las elites mercantiles francesas durante la guerra de Sucesión española / Protecting Business in Time of Crisis: French Trademen during the War of Spanish Succession

\subsection{Maria Fernanda Bicalmo}

Ultramarino y el auge de los secretarios de Estado en Portugal durante la primera mitad del siglo XVIII / Sobre este modo de resolver e despachar os negócios. The decline of the Overseas Council and the Rise of the Secretaries of State in Portugal during the First Half of the $18^{\text {th }}$ Century

\section{9}

\section{VALENTINA FAVARò}

El fracaso de los proyectos de reforma en el virreinato peruano de principios

del siglo XVIII. Las propuestas de Carmine Nicola Caracciolo, príncipe de Santobuono the Eighteenth Century. The Proposals of Carmine Nicola Caracciolo, Prince of Santobuono

\section{7}

\section{ROBERTO QUIRÓS ROSADO}

Ecos de un mercantilismo truncado. El conde de Pinos Puente y la diplomacia comercial de Carlos VI en la corte de Lisboa (1723-1724) / Echoes of a Failed Mercantilism. The Count of Pinos Puente and the Commercial Diplomacy of Charles VI at the Court Of Lisbon (1723-1724)

\section{Junia Ferreira Furtado}

Portuguese America under Foreign Threat and the Creation of the Concept of uti possidetis in the First Half of the $18^{\text {th }}$ Century / La américa portuguesa bajo la amenaza exterior y la creación del concepto de uti possidetis en la primera mitad del siglo XVIII

\section{Miscelánea $\cdot$ Miscellany}

\section{José Antonio Mateos Royo}

con Cataluña / Trade Policy and Monetary Circulation in Aragon: Conflicts and Agreements with Catalonia (1535-1565)

\section{Fernando Altoé}

panegíricos atribuidos a la impresion. Un estudio de la trayectoria de dos the Trajectory of Two Panegyrics Attributed to João de Barros

\section{Francisco Velasco Hernández}

reino de Murcia (siglos XVI y XVII) / The Influence of the Berber Corsican on the Late Repopulation on the Coastal Area on the Kingdom of Murcia (XVI and XVII Centuries)

\section{José Antonio Martínez Martínez}

Criados, jornaleros y esclavos al servicio de la familia: la servidumbre de Serfdom of the Muñoz de Otálora in the $17^{\text {th }}$ Century

\section{Víctor Daniel Regalado González-Serna}

Benito de Medina a raíz de su ingreso en el cabildo catedral de Sevilla en 1669 / «Not a Single Good Portuguese». Accusations against Priest Alonso Benito de Medina when Entering the Cathedral Chapter of Seville in 1669

\section{José Herrera Reviriego}

organigrama comercial y militar de la Gober a tólo mitad del siglo XVII / "Only Time will Tell us»: The Role of Taiwan within the Commercial and Military Organization of the Philippine's Governoration during the First Half of the Seventeenth Century

\section{Manuel-Reyes García Hurtado}

de Rande, 1719-1733 / Vicissitudes of the Rescue Companies of the Sunken Ships in the Battle of Rande, 1719-1733

\subsection{Marcos de Miguel Muñoz}

Caballeros in 1769 


\section{4 ESPACIO, TIEMPO Y FORMA}

\section{Javier Tinoco Domínguez}

Tensiones sociopolíticas en el marco del catastro de Ensenada en Jerez de la Frontera: estudio de un conflicto institucional / Socio-Political Tensions within the Framework of Cadastre of Ensenada In Jerez de la Frontera: A Studying of an Institucional Conflict

\section{Pablo Fernández Albaladejo}

Fábulas de origen y gramática de nación en la España del siglo XVIII. A propósito de algunos trabajos de Francisco Martínez Marina / Origin's Fables and Grammar of Nation in the XVIII Century Spain. About some Works by Francisco Martínez Marina

\subsection{José María IÑURRITEgui Rodríguez}

Constitución increada: Francisco Martínez Marina y la crítica bíblica / Uncreated Constitution. Francisco Martínez Marina and Biblical Criticism

\subsection{David A. Abián Cubillo}

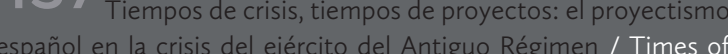
crisis, Times of Projects: The Spanish proyectismo during the Army's Crisis in the Ancient Regime

Taller de historiografía · Historiography Workshop

\section{Ensayos · Essays}

\subsection{ChrISTOPH ROSENMÜLLER}

«Tan peligrosas y feas conspiraciones»: la relación escrita por el embajador austriaco Christoph Migazzi en 1754 sobre la caída del marqués de la Ensenada / «Dangerous and Ugly Conspiracies». The Report of the Austrian Ambassador Christoph Migazzi on the Fall of the Marquis of la Ensenada in 1754

\subsection{Serge Gruzinskı}

Quelle histoire enseigner en 2021 ? / ¿Qué historia enseñar en 2021?

\subsection{Carlos Amate Pizarro}

Las relaciones hispano-chinas en el siglo XVI: síntesis e interpretación a la luz de la reciente historiografía / The Hispanic-chinese Relationship in the XVI Century: Synthesis and Interpretation in the Light of Recent Historiography

\section{Reseñas • Book Review}

521 Bolufer Peruga, Mónica, Arte y artificio de la vida en común. Los modelos de comportamiento y sus tensiones en el Siglo de las Luces, (Julio ArRoyo Vozmediano) 


\section{4 ESPACIO, TIEMPO Y FORMA}

525 Melón, Amando, Alejandro de Humboldt. Vida y obra (Carlos Martínez Shaw)

52 Commentary to Tatiana Seijas' review of The Atlantic World and the Manila Galleons: Circulation, Market, and Consumption of Asian Goods in the Spanish Empire (JosÉ LuIs GASCH TOMAS)

533 Braguier, Laurey, Servantes de dieu. Les beatas de la Couronne de Castille (1450-1600) (Manuela Águeda GARCÍA-GARRIDO)

539 Romeo, María CRuz; SAlomón, María Pilar; TABANERA, Nuria (eds.): Católicos, Reaccionarios y Nacionalistas. Política e identidad nacional en Europa y América Latina Contemporáneas (JAVIER M. Dos SANTOS)

54 Heredia López, Alfonso Jesús, El control de la corrupción en la Monarquía Hispánica. La Casa de la Contratación (1642-1660) (José Manuel Díaz Blanco)

17 Andújar Castillo, Francisco, El Atila de Madrid. La forja de un banquero en la crisis de la monarquía (1685-1715) (Aitor Díaz PAREdes)

551 Serrano Aviles, Javier y Mojarro, Jorge (eds.) Prada GonzALEZ, María (coord. de ilustraciones), En el archipiélago de la Especiería. España y Molucas en los siglos XVI y XVII (İ̃̃ıGo VALPUESTA VILLA)

555 Díaz Ceballos, Jorge, Poder compartido. Repúblicas urbanas, Monarquía y conversación en Castilla del Oro, 1508-1573 (JUAN SEbAstián Gómez GonZÁleZ)

51 Edelmayer, Friedrich, Massimiliano II, Filippo II I'Italia imperiale. II marchesato di Finale, i diritti imperiali e il «camino spagnolo» (RAFAEL VALLADARES)

56 Escribano-PÁEZ, José M., Juan Rena and the Frontiers of Spanish Empire, 1500-1540 (DAvid Martín Marcos)

56 ARnOLD, David, La Era de los Descubrimientos (1400-1600), Madrid, Alianza Editorial, 2021, 184 Pp., ISBN: 978-841362-172-2 (CARlos Amate Pizarro) 